\title{
Epidemiological Investigation of Renal Colic and Research on Intervention Measures in a Field Training Troop in Northwest China
}

\author{
Ding Yancai ${ }^{\dagger}$, Liu Li $^{\dagger}$, Li Feiyu ${ }^{*}$, Yang Tao, Ma Xiaoyun, Xi Haifeng \\ Department of Urology, 942 Hospital of PLA, Yinchuan, China \\ Email address: \\ 824798002@qq.com (Li Feiyu) \\ ${ }^{*}$ Corresponding author \\ $\dagger$ Ding Yancai and Liu Li are co-first authors.
}

\section{To cite this article:}

Ding Yancai, Liu Li, Li Feiyu, Yang Tao, Ma Xiaoyun, Xi Haifeng. Epidemiological Investigation of Renal Colic and Research on Intervention Measures in a Field Training Troop in Northwest China. Clinical Medicine Research. Vol. 10, No. 5, 2021, pp. 164-168.

doi: $10.11648 /$ j.cmr.20211005.12

Received: September 30, 2021; Accepted: October 25, 2021; Published: October 29, 2021

\begin{abstract}
Background: To understand the incidence of renal colic and pain index scores during field training (summer and autumn) of military personnel, and to formulate intervention measures. Methods: A total of 3,856 people were surveyed in the three foreign training years from 2016 to 2018. An epidemiological survey was conducted on the training time of foreign training troops in summer and autumn, the training environment, temperature changes, dietary structure, age, gender, water consumption, etc.; at the same time; For soldiers with renal colic caused by urolithiasis, the blood uric acid level, blood phosphorus, blood calcium and other biochemical indicators were detected, intervention treatment was carried out, and the stone specimens produced after the treatment were analyzed for the stone composition. Results: 49 cases of renal colic occurred in 3856 people. The average incidence of renal colic was $1.2 \%$, and the visual analog score index of renal colic was average (8.2 \pm 1.8$)$. Among them, 47 were males and 2 were females. The average age was $(22 \pm 2.5)$ years, the average stone size was $(0.6 \pm 0.2) \mathrm{cm}$, the average temperature was $(38.5 \pm 7.2)^{\circ} \mathrm{C}$, and the average water consumption was $(2000 \pm 300) \mathrm{mL}$. In 2016, there were 17 cases of 1150 cases, and the incidence of renal colic was $1.4 \%$; in 2017,12 cases of 1,320 cases, the incidence of renal colic was $0.9 \%$; in 2018,14 cases of 1386 cases, the incidence of renal colic, $1.1 \%$. The average uric acid level was $(282 \pm 11) \mathrm{mmol} / \mathrm{L}$, the average blood phosphorus $(0.98 \pm 0.03) \mathrm{mmol} / \mathrm{L}$, and the average blood calcium $(2.63 \pm 0.08) \mathrm{mmol} / \mathrm{L} .26$ cases were cured by conservative treatment, 19 cases were cured by extracorporeal shock wave lithotripsy, and 4 cases were cured by ureteroscopy. Urinary stones are mainly composed of monohydrate and dihydrate calcium oxalate stones. Conclusion: The incidence of renal colic in the field stationed troops in the northwest arid area in summer and autumn and high temperature environment is mainly caused by ureteral stones. The symptoms can be relieved by conservative treatment such as spasmolysis, analgesia, and stone removal; conservative treatment is the main treatment method, ureteroscopy Treatment assistance, no obvious adverse events occurred.
\end{abstract}

Keywords: Renal Colic, Epidemiological Investigation, Intervention Measures

\section{Introduction}

Urinary calculi is a common disease in urology, and its specific mechanism and related risk factors are still unclear. It is generally believed that age, gender, race, genetics, environmental factors, eating habits, and occupation are related to the formation of stones [1-2]. Clinically, renal colic is often the main first symptom, which has the characteristics of rapid onset and severe illness. Because of the high incidence of renal colic in soldiers, it is a group of important diseases that cause non-combat attrition [3]. Some domestic scholars have made relevant investigations and studies on the incidence of kidney stones and acute abdomen in hospitals in military garrison [4]. There is currently no research report on the epidemiological investigation of renal colic and preventive intervention measures during military field training. Therefore, 
this study The epidemiological survey of renal colic in military personnel in foreign training troops, to understand the incidence of renal colic in foreign training troops, to improve rapid diagnosis and to study reasonable emergency treatment methods, is now reported as follows.

\section{Materials and Methods}

\subsection{General Information}

An epidemiological survey was conducted on a total of 3,856 people in a training base in Northwest China during the three foreign training years (July-September in summer and autumn) from 2016 to 2018 . Among them, 1,875 were soldiers over 2 years, 786 were over 5 years, 912 were over 8 years, and 283 were over 12 years.

\subsection{Method}

According to the method of epidemiological survey and statistics, the survey form was developed by 2 attending urological surgeons to conduct the survey, including training time, training environment, temperature change, diet structure, age, gender, education level, water consumption, etc. Renal colic is diagnosed by urinary system color Doppler ultrasound or three-dimensional reconstruction CT scan of the kidney and ureter. For soldiers with renal colic caused by urolithiasis, the blood uric acid, blood phosphorus, blood calcium and other biochemical indicators are tested in our hospital for intervention and treatment. Afterwards, the stone specimens were analyzed for the composition of the stones. The collected stone specimens are dried, crushed into powder, potassium bromide is added, and the tablets are pressed for infrared spectroscopy. The stone infrared spectroscopy automatic analysis system produced by Tianjin Lanmod Scientific Instrument Co., Ltd. is used to analyze the stone composition.

\subsection{Statistical Analysis}

The SPSS 17.0 software package is used for data analysis. Measurement data are expressed as mean \pm standard deviation ( $\bar{x} \pm s$ ), using 2 test; counting data are expressed as number of cases (percentage) $[\mathrm{n}(\%)]$, using t test. The difference is statistically significant when $\mathrm{P} \leq 0.05$.

\section{Result}

\subsection{The Occurrence of Renal Colic}

There were 49 cases of renal colic in 3856 people, the average incidence of renal colic was $1.2 \%$, the average stone size was $(0.6 \pm 0.2) \mathrm{cm}$, and the average renal colic visual analog score index was ( $8.2 \pm 1.8)$. Among them, 47 were males. There were 2 females, the average age was $(22 \pm 2.5)$ years; the average temperature was $(38.5 \pm 7.2)^{\circ} \mathrm{C}$, and the average water consumption was $(2000 \pm 300) \mathrm{mL}$. See Table 1.

Table 1. The basic situation of the personnel in the field training troops and the occurrence of renal colic $(n=3856)$.

\begin{tabular}{|c|c|c|c|c|}
\hline relevant factor & $n$ & occurrence of renal colic n (\%) & $\chi^{2}$ value & $P$ value \\
\hline Age & & & 12.83 & 0.073 \\
\hline $20 \sim 24$ & 1875 & $27(1.3)$ & & \\
\hline $25 \sim 30$ & 786 & $8(0.8)$ & & \\
\hline$>30$ & 1195 & $14(0.9)$ & & \\
\hline \multicolumn{5}{|l|}{$\operatorname{sex}$} \\
\hline male & 3812 & $47(1.2)$ & & \\
\hline female & 44 & $2(0.4)$ & & \\
\hline Education & & & 46.15 & 0.021 \\
\hline Bachelor degree and above & 428 & $3(0.6)$ & & \\
\hline Undergraduate & 3428 & $46(1.3)$ & & \\
\hline Soldier age & & & 13.28 & 0.073 \\
\hline$>2$ years $<8$ years & 2661 & $36(1.3)$ & & \\
\hline 9 years $<12$ years & 912 & $10(1.1)$ & & \\
\hline$>12$ years & 283 & $3(0.9)$ & & \\
\hline 2016 & 1150 & $19(1.4)$ & & \\
\hline 2017 & 1320 & $13(1.1)$ & & \\
\hline 2018 & 1386 & $18(1.3)$ & & \\
\hline
\end{tabular}

\subsection{Changes of Blood Uric Acid, Blood Phosphorus and Blood Calcium Indexes Before and After Treatment}

There was no statistically significant difference in the average uric acid level and blood phosphorus level before and after treatment $(\mathrm{P}>0.05)$, and the difference in the average blood calcium level was statistically significant $(\mathrm{P}<0.05)$, see Table 2 .

Table 2. Changes of blood uric acid, blood phosphorus and blood calcium indexes before and after treatment of renal colic $(n=49, \bar{x} \pm s, m m o l / L)$.

\begin{tabular}{llll}
\hline time & Blood uric acid & Blood phosphorus & Blood calcium \\
\hline before therapy & $282 \pm 11$ & $0.98 \pm 0.03$ & $2.63 \pm 0.08^{*}$ \\
after therapy & $252 \pm 19$ & $1.02 \pm 0.05$ & $1.98 \pm 0.05$ \\
\hline
\end{tabular}




\subsection{Treatment Status and Analysis of Stone Composition}

26 cases were treated conservatively by antispasmodic (phloroglucinol, Nospar), analgesia (pethidine or morphine, nimesulide dispersible tablets), Paishi (tamsulosin sustained-release capsules, Wulinhuashi pills) Healed, 19 cases underwent extracorporeal shock wave lithotripsy, and 4 cases underwent ureteroscopic holmium laser lithotripsy under general anesthesia. Thirty-eight cases of stone samples were collected. The urinary stones were mainly calcium oxalate monohydrate and dihydrate stones, as shown in Table 3.

Table 3. The urinary stones were mainly calcium oxalate monohydrate and dihydrate stones.

\begin{tabular}{llll}
\hline Stone composition & Kidney and upper ureter $(\boldsymbol{n})$ & Lower ureter $(\boldsymbol{n})$ & sum $[\boldsymbol{n}(\%)]$ \\
\hline Calcium oxalate monohydrate & 4 & 5 & $9(23.6)$ \\
Calcium oxalate dihydrate & 4 & 14 & $19(47.3)$ \\
Monohydrate + dihydrate calcium oxalate & 3 & 8 & $11(28.9)$ \\
\hline
\end{tabular}

\section{Discussion}

The natural environment in the northwestern region is harsh, with high temperature difference between day and night and high ultraviolet intensity. Under such an environment, the incidence of urinary calculi is relatively high [4-5]. Urinary calculi can cause symptoms such as acute pain, hematuria, fever, and other adverse consequences such as acute and chronic renal insufficiency, hydronephrosis, loss of renal function, and even nephrectomy in severe cases. The sudden renal colic in soldiers during external training causes physical and psychological changes, which seriously affects combat effectiveness and physical health. How to prevent the onset of renal colic, early diagnosis and active intervention has become an important mission that frontline military doctors urgently need to solve. The high incidence of urinary calculi is 22 to 55 years old (approximately 84.3\%), which may be related to natural environment, social living conditions, metabolic disorders, occupational and urinary system disorders [6]. Zeng Guohua et al. [7] conducted a cross-sectional survey on the prevalence of urolithiasis among adults in 7 domestic provinces and municipalities directly under the Central Government. The results showed that the average prevalence rate of urolithiasis among adults in my country was $6.06 \%$, the highest in Guangdong (11.63\%). And men are higher than women, rural areas are higher than cities, and the south is higher than the north. Forces are usually located in remote areas. During the summer and autumn training, the weather is dry, the training conditions are difficult, the diet structure is unreasonable, the intensity of military training, sweating and insufficient drinking water are the main reasons for the occurrence of urinary calculi; Young males are also a high-risk group of urinary calculi, which is the main cause of non-combat attrition in the army [8].

This study found that the incidence of renal colic caused by urinary calculi during external training was $1.2 \%$ on average, mainly young people, with an average age of about 20 years (may be related to the age distribution characteristics of the study population), and the incidence of renal colic in female soldiers It is $0.4 \%$, and the incidence is low. Because the external training is mostly summer and autumn, the northwestern region has hot and dry climate, large temperature difference between day and night, large amount of training, insufficient drinking water, and unreasonable dietary structure are the main causes of the disease. Urinary stones are the main part of the affected population, and the lower ureteral stones are especially frequent. The size of the stones is about $0.6-0.8$ $\mathrm{cm}$. The uric acid level is generally low, but the blood calcium level is higher than that of the normal population. The urinary stones are composed of monohydrate and dihydrate. Calcium oxalate is predominant; in patients with renal colic, the visual analogue score of renal colic is above 8 points, indicating that the symptoms are obvious, and emergency intervention is required. Therefore, considering that the above-mentioned stones are mostly formed suddenly, the stones are small in size, the process of stone removal is rapid, and the stones are stuck in the ureteral stenosis, resulting in acute renal colic attacks and severe symptoms; and the patients are mostly young soldiers, and the pain reaction is generally sensitive. Front-line military doctors are often helpless due to lack of understanding. Patients need to be accompanied by multiple people to evacuate them for emergency treatment, resulting in non-combat attrition and psychological stress in their teams, which will affect follow-up training in the short term. It is particularly urgent to improve the level of diagnosis and treatment of the disease by frontline military doctors, as well as the health education and psychological comfort of patients. In this study, conservative treatment was the main treatment method, and no obvious adverse events occurred.

The author believes that strengthening the epidemiological research on the causes of urinary calculi in military personnel in training bases, and understanding its occurrence, development and outcome, can formulate effective prevention and treatment strategies for front-line troops and provide scientific and reasonable diagnosis and treatment decisions for front-line military doctors, thereby reducing non-compliance. Fighting attrition and reducing the aggravation of symptoms caused by patients' psychological panic are of great significance. In the prevention and treatment strategy, the following points should be paid attention to: (1) Drink more water. The daily water consumption of external trainers should be more than $3000 \mathrm{~mL}$ in order to effectively increase the urine output. The urine color is used to initially judge whether the water consumption is sufficient, and the urine color is light yellow. It is suggested that adequate drinking water can reduce the saturation and timely excretion of various stone-forming substances in the urine; studies have shown that the incidence 
of urinary stones is $3.4 \%$ for those who drink $2000-3000 \mathrm{~mL}$ daily, and the daily drinking water is more than $3000 \mathrm{~mL}$ Patients, the incidence rate is $0.9 \%$, when the daily drinking water is greater than $3000 \mathrm{~mL}$, it can prevent the occurrence of urinary calculi [9]. (2) Reasonable dietary structure, reduce the intake of excessive animal protein. Excessive intake of animal protein will increase the concentration of oxalic acid in the urine, reduce the $\mathrm{pH}$ value of the urine, and promote the formation of uric acid-based stones; at the same time increase plant fiber The intake of citrus can promote intestinal peristalsis, regulate intestinal flora, reduce intestinal hyperoxaluria, and prevent the occurrence of stones; eat citrus regularly, because citrus fruits can increase the content of citrate (calculus inhibitor) in urine, Helps prevent the recurrence of stones [10]. (3) Intake of proper amount of calcium will help prevent the occurrence of urinary calculi. Calcium in food combines with oxalic acid in the intestine to form a complex. Low calcium leads to an increase in oxalic acid absorption and increases the risk of oxalate stone formation. High calcium leads to an increase in the concentration of calcium in the urine, which increases the risk of calcium as the main component of stone formation [12]. At present, the recommended daily calcium intake for patients with urinary tract stones is $800-1200 \mathrm{mg}$ [11].

For first-line military doctors to pay attention to the following points in diagnosis and treatment decisions: (1) Improve the ability to distinguish acute abdomen, urinary calculi often have sudden onset of back pain, hematuria, with or without nausea and vomiting, passive position (backward position), Pain does not decrease with changes in body position, some have radiating pain in the lower abdomen, scrotum, urinary tract irritation, no signs of peritoneal irritation, no factors that induce gastrointestinal diseases (such as unclean diet), no diarrhea, no metastatic lower abdominal pain; The typical clinical manifestations are mainly paroxysmal, radiation along the ureteral path, severe and unbearable pain, often accompanied by nausea, vomiting or oliguria. (2) After the diagnosis is clear or highly suspected and other acute abdomen is ruled out, antispasmodic (phloroglucinol, Nospar, etc.), analgesia (pethidine or morphine), rehydration (about $2000 \mathrm{~mL}$ ) and other symptomatic treatments are given, and the changes in the condition are observed Most of the symptoms can be relieved after the above treatment, and some small ureteral stones can be discharged on their own, so there is no need to worry too much, and serious complications will not occur in the short term; if the symptoms continue to worsen, rush to the system military hospital or the nearest hospital Diagnosis and treatment.

In short, the exact cause of urolithiasis is still unclear, and there is no ideal preventive measure. The contradiction between daily training, water consumption, dietary structure, etc. cannot be fully reconciled during foreign training. Sudden renal colic is restricted by the site and environment, and cannot be diagnosed and treated in time. How to provide personalized prevention and treatment measures for military stone patients to effectively reduce the incidence of stones is still the direction of military hospitals for the prevention and treatment of urinary calculi [13-14].

\section{Conclusion}

The incidence of renal colic in the field stationed troops in the northwest arid area in summer and autumn and high temperature environment is mainly caused by ureteral stones The symptoms can be relieved by conservative treatment such as spasmolysis, analgesia, and stone removal; conservative treatment is the main treatment method, ureteroscopy Treatment assistance, no obvious adverse events occurred [15].

\section{References}

[1] Zhang Hanrong, Gao Qinghe, Yang Zhenyou, et al. Meta-analysis of risk factors related to urinary calculi in Chinese adults [J]. Chinese Journal of Urology, 2018, 39 (12): 935-939.

[2] Guillén R, Ramos C, Ayala R, et al. Lithogenic risk index in urinary lithiasis patients and their evolution after treatment $[\mathrm{J}]$. Arch Esp Urol, 2017, 70 (8): 725-731.

[3] Guo Shujun, Wang Jinhui, Lu Jinli, etc. Analysis of common surgical acute abdomen in soldiers [J]. Clinical Journal of Military Medicine, 2008, 36 (1): 130.

[4] Xu Li, Shi Runze, Wu Lin, et al. Investigation and analysis of the disease spectrum of hospital officers and soldiers in a hospital in the plateau of Northwest China [J]. Chinese Journal of Preventive Medicine, 2018, 36 (11): 1469-1486.

[5] Yin Xudong, Lu Lin, Tang Bin, et al. Analysis of the main diseases composition of summer plateau training for non-plateau troops and plateau troops [J]. Chinese Journal of Preventive Medicine, 2014, 32 (2): 110.

[6] Lin Feihe, Xie Chunfa, Gao Hailiang, et al. Clinical analysis of 792 cases of urinary calculi [J]. Hainan Medicine, 2010, 21 (7): 80-81.

[7] Zeng Guohua, Mai Zanlin, Xia Shujie, et al. Cross-sectional survey on the prevalence of urolithiasis in Chinese adults [J]. Chinese Journal of Urology, 2015, 36 (7): 528-532.

[8] Zhang Qian, Chen Yudong, Han Yao, et al. Analysis and clinical significance of 236 cases of urinary calculi in garrison soldiers [J]. Medical Journal of Chinese People's Liberation Army, 2013, 25 (2): 75-76.

[9] Ye Ailan, Zhou Fengchang, Cai Xianqiu, et al. Investigation and analysis of the incidence of drinking water and urinary calculi (with a report of 1431 cases) [J]. Chinese Journal of Modern Surgery, 2005, 2 (6): 576-578.

[10] Tian Jing, Guo Hongqian, Sun Xizhao, et al. Preparation and evaluation of a diet-related etiology model of calcium oxalate stones in rats [J]. Journal of Medical Postgraduates, 2012, 25 (5): 555-558.

[11] Liu Jun, Li Songlin, Tai Changsong, et al. A cross-sectional survey of kidney stones and dietary factors [J]. Modern Preventive Medicine, 2002, 29 (2): 168-170. 
[12] Liu Xin, Su Hongwei, Chen Jie, et al. The mechanism of Tamm-Horsfall protein on kidney stone formation [J]. Journal of Medical Postgraduates, 2017, 30 (9): 922-925.

[13] Lin Yudong, Wu Dezhu, Wang Jianping, et al. Clinical study of 238 cases of urolithiasis in troops stationed in a certain area in the southeast $[\mathrm{J}]$. Southeastern National Defense Medicine, 2013, 15 (3): 219-220.

[14] Jin Lei, Xu Binxian, Xiang Junji, et al. Investigation and analysis of related factors of urinary calculi in a flying unit stationed in Jiangsu [J]. Southeast National Defense Medicine, 2014, 16 (5): 529-530.

[15] Zhang Tao, Ding Yancai, Jiang Xu, et al. Minimally invasive percutaneous nephroscope holmium laser combined with pneumatic lithotripsy for the treatment of kidney and upper ureteral calculi (report of 1870 cases) [J]. Chinese Journal of Minimally Invasive Surgery, 2013, 13 (06): 513-515. 